\title{
To build better tuberculosis diagnostics, look for 'biosignatures'
}

As many as a third of individuals with HIV simultaneously suffer from tuberculosis. At the Conference on Retroviruses and Opportunistic Infections held in Seattle earlier this year, scientists emphasized routine and rapid diagnostics that could test not only whether tuberculosis was present but also whether the pathogen showed resistance to currently available drugs.

The need for improved diagnostic tests for tuberculosis continues to intensify. Currently, when an individual shows symptoms that point to tuberculosis infection, he or she is sometimes placed in respiratory isolation while awaiting results from a microscopy test that looks for tuberculosis bacteria in sputum. This testing protocol, called a sputum test, should ideally be a one- to two-day process; it can, however, take longer. "I've seen patients be isolated for as long as a week, and this has happened over and over again," says David Alland, professor of medicine at Rutgers University.

Growing the samples in a Petri dish or test tube provides greater sensitivity when testing for tuberculosis. Immunocompromised individuals, such as those with HIV, especially benefit from this method, as they seldom produce lung cavities. Lung cavities are produced as a result of the large antigenic load during the course of tuberculosis infection, but in people with weakened immune systems, these formations are often missing. The cavities hold large amounts of tuberculosis bacteria, which are detectable when the patient coughs up a sample for a sputum test, but the lack of cavities in some individuals means fewer detectable bacteria during testing. One catch, though, is that culture tests require sophisticated handling and proper storage to prevent contamination, and they can take at least two weeks to produce a result. Especially in the developing world, where HIV and tuberculosis co-infection is growing, the need for quicker tests is dire.

Marc Nicol, principal investigator in the department of clinical laboratory sciences at the University of Cape Town, South Africa, explains: "In our setting it's difficult to get patients back two weeks later, [so] often that culture result will just sit on a clinic disk and nobody does anything about it."

\section{Signature approach}

Some researchers are trying to close the diagnostics gap by creating a 'biosignature' for the detection of tuberculosis. Current diagnostics look for single markers such as the presence of the tuberculosis pathogen, but researchers are trying to develop ways of detecting multiple markers at the same time. In one review of current attempts to create a platform that detects multiple markers, the authors found that platforms that detect transcriptional activity in tuberculosis can be up to $95 \%$ accurate, but added that other types of platforms are still needed to create a uniform biosignature for the disease (Cold Spring Harb. Perspect. Med., 5, a018531, 2015).

Other groups are focusing on gene expression signatures of this illness. For example, a study of about 3,000 children in South Africa, Malawi and Kenya identified a promising 51-transcript RNA expression signature associated with tuberculosis (N. Engl. J. Med. 370, 1712-1723, 2014). Another paper published last year found an expression signature of 251 genes that could detect tuberculosis in patients with and without HIV co-infection (PLoS ONE, doi: 10.1371/journal.pone.0089925, 2014). Just this past May, a study described a new, real-time PCR signature that the authors believe can distinguish between tuberculosis and other pulmonary diseases (Tuberculosis, doi:10.1016/j.tube.2015.04.008, 2015).

Meanwhile, in response to the growing threat of multidrug resistant tuberculosis (MDR-TB), diagnostics research and development has looked beyond simply testing for the presence of tuberculosis. In 2011, Alland and his colleagues developed the Xpert MTB/RIF system, in collaboration with Cepheid in Sunnyvale, California. It's a test that uses DNA markers to simultaneously identify the presence of the tuberculosis bacterium (Mycobacterium tuberculosis, MTB) and whether it carries known genetic markers for resistance to rifampin (RIF), a first-line antibiotic against the disease. "The core of the [Xpert] assay is a real-time PCR assay," says Nicol, who has led field trials of the system in South Africa. "It's essentially entirely automated," making it suitable for low-resource settings.

Although experts laud Xpert for its speed and sensitivity, at least one study found that its false-positive rate for drug resistance was more than 30\% (Diagn. Microbiol. Infect. Dis., 74, 207-209, 2012). Xpert also suffers from limited sensitivity in the growing population of HIV-positive tuberculosis patients, a point that Alland concedes. Continuing its collaboration with the researchers who developed the original Xpert assay, Cepheid hopes to address these problems with an improved test, provisionally named Xpert Ultra. That system is now entering human trials.

\section{The MODS squad}

Some scientists wonder whether, while testing for RIF resistance, it would also be worthwhile to test for resistance to isoniazid (INH), the other first-line antibiotic against tuberculosis. One test that looks at resistance to INH is the microscopic observation drug susceptibility (MODS) assay, which combines two traditional phenotypic tests, microscopy and sputum culture. The test works by culturing a sputum sample in liquid medium and then examining the culture under a microscope to detect the bacteria as soon as they start to grow. Researchers at the Universidad Peruana Cayetano Heredia in Lima, Peru, originally developed the technique, which has since been refined and tested in the field.

"It's just as sensitive as culture, because it is culture," says Sarita Shah, a scientist within the division of tuberculosis elimination at the US Centers for Disease Control and Prevention in Atlanta. Shah's team tested MODS in a rural hospital in South Africa, and they found that the assay was highly sensitive and specific, even in patients co-infected with HIV and tuberculosis (Am. J. Respir. Crit. Care Med., 183, 1427-1433, 2011).

Others suggest, however, that adding a testing component for INH resistance would not necessarily address the problem of MDR-TB. For instance, one study that used a transmission model to simultaneously test for INH resistance and RIF resistance found that the prevalence of MDR-TB was reduced by only $4 \%$ compared using only a rapid test for tuberculosis that assessed RIF resistance (PLoS ONE, 9, e84197, 2014).

At present, nearly one-third of the people in the world are infected with the tuberculosis bacterium. Despite the move toward improved tuberculosis diagnostics, any good test needs good implementation on the ground, says Mark Perkins, chief scientific officer of the Foundation for Innovative New Diagnostics in Geneva. "A diagnostic is not an intervention, it's the start of an intervention."

Alan Dove 